\title{
Guest Comment: Set Theoretic Concepts in Computer Science and Engineering-A Connection between Mathematics and Computing
}

\author{
Dele Oluwade \\ College of Science \& Technology \\ P.O. Box 20253 \\ University of Ibadan \\ Ibadan, Oyo 200005 NIGERIA
}

The well-known intuitive definition of a set is any well-defined collection of objects. Sets arise naturally in nature and are an important part of a student's curriculum, directly or indirectly, from elementary school. The theory pervades all areas of science and other fields of human endeavor.

Traditionally, there are two approaches to set theory namely the intuitive approach and the axiomatic approach. Intuitive set theory was developed by Cantor in the 1880s. This theory was however heavily criticized by

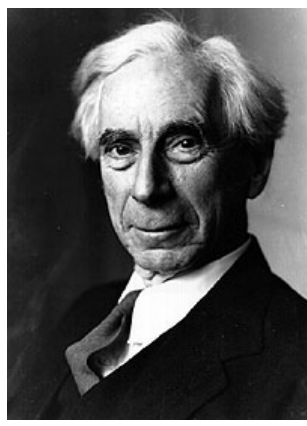

Bertrand Russell
Bertrand

Russell (1872-1970). Russell exhibited a set which doesn't contain itself as elements-the socalled Russell set. The axiomatic approach is much deeper with logical technicalities than the intuitive approach. Apart from Russell, other notable contributors to the theory include Zermelo, Fraenkel, Godel, Cantor, Zorn, Cohen, De Morgan, Euler, Venn, Descartes, Schroeder, Bernstein, Peano, Hilbert, Boole and Frege.

The occurrence and application of the notion of sets abound in digital computing. Such sets include instruction set, code, coded character set, recursive set and recursively enumerable set. . The Russell set (or Russell paradox) played an important role in the evolution of the theory of computation. (It is well-known that the answer as to whether this set is an element of itself is neither yes nor no!) Internally, sets occur in a digital computer system as a discrete phenomenon due to the fact that it is ordinarily a Boolean system. In the design of the programming

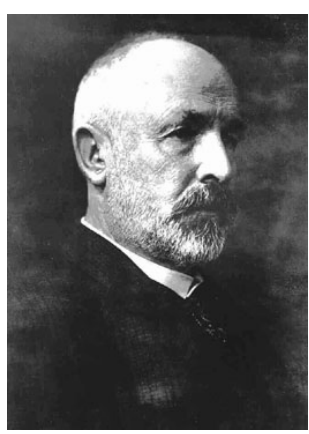

Georg Cantor language 'LEAP', sets of triples were used to organize a database in the design.

Computer science, which presently has its distinct identity, was historically an offshoot of mathematics. For instance, the widely recognized father of computing,

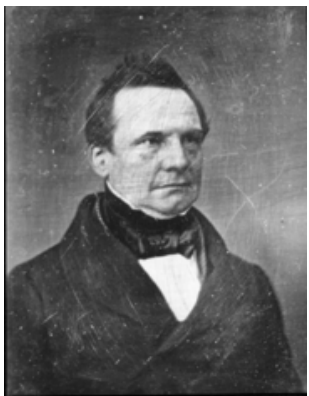

Charles Babbage (ca. 1843) Charles Babbage, was a professor of mathematics. Other great mathematicians such as Alan Turing and John von Neumann played a key role in the development of the stored program computers. It would be to the long term benefits of both mathematics and computing to maintain their close relationship while still preserving their individual identities. Coding theory, in particular, provides a unifying platform for both mathematics and computer science \& engineering. 